\title{
Effect of Sildenafil on Mortality in Term Neonates with Hypoxemic Respiratory Failure due to Persistent Pulmonary Hypertension (SIPHON) - A Randomised Control Trial
}

\section{Deepti Thandaveshwara, Sushma Krishnegowda, Divya Hosur and Srinivasa Murthy Doreswamy}

Department of Paediatrics, JSS Medical College, JSS Academy of Higher Education and Research, Mysuru, Karnataka, India

\author{
Correspondence: \\ Srinivasa Murthy Doreswamy \\ Department of Paediatrics, \\ JSS Medical College, \\ JSS Academy of Higher Education and \\ Research, \\ Mysuru, Karnataka, India \\ Email: drdsrinivasa@gmail.com
}

DOI: $10.3126 /$ jnps.v41i2.32403

Submitted on: $2020-10-21$

Accepted on: 2021-06-30

Acknowledgements: None

Funding: Nil

Conflict of Interest: None declared

Permission from IRB: Yes

To cite this article: Thandaveshwara $\mathrm{D}$, Krishnegowda S, Hosur D, Doreswamy SM. Effect of Sildenafil on Mortality in Term Neonates with Hypoxemic Respiratory Failure due to Persistent Pulmonary Hypertension (SIPHON) - A Randomised Control Trial. J Nepal Paediatr Soc. 2021;41(2):177-83.

\begin{abstract}
Introduction: Persistent Pulmonary Hypertension (PPHN) complicates the transition from intrauterine to extrauterine life in some neonates. Sildenafil is known to reduce pulmonary pressures. However, its effect on reducing mortality is unclear. This study is conducted to determine the effect of Sildenafil in reducing all-cause mortality rate in term newborns with hypoxemic respiratory failure due to pulmonary hypertension.

Methods: This was a double-blinded randomised control trial done in a level 3 neonatal intensive care unit. Term neonates on mechanical ventilation with an Oxygenation Index $(\mathrm{OI})>15$, and echocardiography suggesting PPHN were included in the study. The drug group received intravenous Sildenafil and the placebo group normal saline. The difference in the mortality rate between the two groups was analysed.
\end{abstract}

Results: Eleven babies were randomised to the drug group and 12 to the placebo group. Both the groups matched in terms of their baseline characteristics. The difference in the drop of OI and right ventricular systolic pressure (RVSP) between the drug and placebo groups was not statistically significant. The relative risk of mortality was 0.436 in the intervention group (95\% CI 0.105 to $1.80)$. The difference in the mortality rate was not statistically significant with P-value of 0.252 . The rate of complications such as necrotising enterocolitis, sepsis, and pneumothorax was not different between the two groups.

Conclusions: Intravenous sildenafil does not have any significant benefit over placebo in reducing all-cause mortality, oxygenation index, RVSP or complications when compared to placebo.

Keywords: Hypertension; Mortality; Neonates; Sildenafil; Ventilation

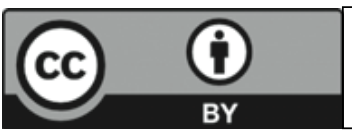

This work is licensed under creative common attribution 3.0 license 


\section{INTRODUCTION}

Successful transition of neonates to extrauterine life involves a decrease in the pulmonary vascular pressure facilitating optimisation of ventilation perfusion ratio. On occasions, if this fails to occur, pulmonary vascular pressure remains high and leads to undesirable intrapulmonary shunting leading to resistant hypoxia. This condition is known as persistent pulmonary hypertension of the neonate (PPHN) characterised by increased pulmonary vascular resistance (PVR), right-to-left shunting, and severe hypoxemia. ${ }^{1}$ The etiology can be multiple including meconium aspiration, pneumonia, sepsis, lung hypoplasia, alveolar capillary dysplasia but often could be idiopathic. ${ }^{1}$ Its incidence has been reported as 1.9 per 1000 live births with a mortality rate ranging between 4 $33 \%{ }^{2}$ In Asian countries, the incidence ranged from 1.2 to 4.6 per 1000 live birth as reported in a multi-centric trial. ${ }^{3}$ The diagnosis of PPHN is based on clinical evidence of labile hypoxemia often associated with differential cyanosis. The diagnosis is confirmed by the echocardiographic demonstration of (a) right-to-left or bidirectional shunt at the ductal and/or oval foramen and / or, (b) flattening or leftward deviation of the interventricular septum and / or, (c) tricuspid regurgitation, in the absence of structural heart disease. ${ }^{4}$ Gentle ventilation strategies, lung recruitment, inhaled nitric oxide (iNO), and surfactant therapy have been used with promising results. ${ }^{5}$ Inhaled nitric oxide is an expensive and labor - intensive intervention that is rarely available in many resource - limited settings. A failure rate of $30 \%$ is reported in the literature. ${ }^{6}$ Even with appropriate therapy, the mortality for PPHN remains between $5-10 \%{ }^{7}$

Sildenafil is a phosphodiesterase inhibitor type 5 (PDE5) that has shown to selectively reduce PVR in both animal models and adult humans. ${ }^{8}$ But its effect on reducing the mortality in PPHN is yet to be ascertained. There is also limited data suggesting the role of intravenous Sildenafil in ventilated babies. It is currently used as an adjuvant in iNOresistant PPHN and as an acute primary treatment of PPHN when iNO is not available or contraindicated. ${ }^{9}$

We undertook this study to know the effectiveness of IV Sildenafil in term newborns with PPHN. The primary objective was to determine the effects of Sildenafil treatment on the all-cause mortality rate in term newborns with hypoxemic respiratory failure due to PPHN. The effects of Sildenafil treatment on duration of mechanical ventilation in term newborns and the effect on pulmonary pressures over 72 hours of treatment were also studied.

\section{METHODS}

This was a double-blinded randomised control trial with the intention to treat analysis conducted in a tertiary neonatal intensive care unit. The study was initially planned to be conducted over 18 months. However as only a few subjects were recruited, the study period was extended for another 18 months. Term neonates on mechanical ventilation with hypoxemic respiratory failure (Oxygenation index $>15$ ) and if fulfilling at least two of the following four findings on ECHO like - a) Right Ventricular Systolic Pressure (RVSP) > $40 \mathrm{mmHg}$, b) RVSP / systemic systolic blood pressure $>0.5$, c) Any cardiac shunt with bidirectional or right to left flow and d) Any degree of ventricular septal flattening was included in the study. Babies with congenital diaphragmatic hernia, surgical lung anomalies or severe external congenital anomalies were excluded. We planned the study of independent cases and controls with one control (s) per case. Prior data from our unit indicate that the mortality rate among severe PPHN neonates is $50 \%$. We assumed a relative risk in the intervention group of $10 \%$, alfa of $5 \%$, and a power of $80 \%$. Hence, we needed a sample size of 15 in each group. Simple random sampling was adopted for this study. Randomisation was done as per the random numbers generated in computers. The allocation was concealed in an opaque envelope and opened at the time of assigning the recruited subjects. Blinding of the medication was done using a saline placebo packed in similar - looking syringes. Both Sildenafil and saline syringes were coded and later decoded for analysis (Figure 1).

Study subjects randomised to the study group were treated with Sildenafil citrate (Pulmosil, Sun Pharma, India - $10 \mathrm{mg} / 12.5 \mathrm{ml}$ ). Sildenafil was initially given as IV bolus of $1.2 \mathrm{ml} / \mathrm{kg} /$ dose followed by $0.6 \mathrm{ml} / \mathrm{kg} /$ dose six hourly for the next 72 hours along with other standard treatment as per our unit protocol. Study subjects randomised 
to the control group were administered normal

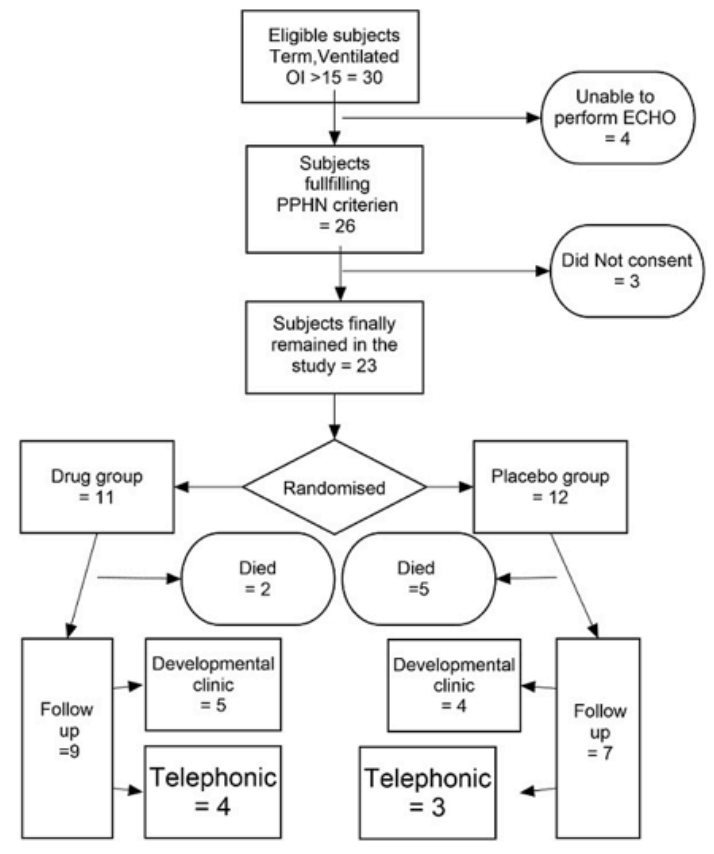

Figure 1. CONSORT diagram

saline along with other standard treatment as per our unit protocol. ECHO was done using Philips CX 30 (Singapore) machine and a sector probe at a frequency of $10 \mathrm{MHz}$. Echo was done by the Echo technician at the bedside and the images were interpreted by the cardiologist. All the babies were ventilated on Macquet servo I Neonatal (Sweden) on pressure control mode. SIMV with pressure support was used for weaning from the ventilator. All the neonates who survived were followed up periodically in neuro - developmental follow-up clinic up to the age of two years. For babies who were unable to attend the clinic at their second birthday, parents were contacted over the phone to know the status of their children. Institutional Ethical board approved this study (JSS/MC/IEC/ 02/661/2015-16). Informed consent was taken from parents or immediate caregivers. The trial was registered under the Clinical Trials Registry of India (CTRI/2017/11/010688). The proportion of babies who died in each group was compared using a chi-square test. Relative risk, with 95\% CI and number needed to treat was reported. The median duration of respiratory support days between the two groups was compared using a sign test. P value of $<0.05$ was considered as statistically significant. Statistical analysis was done using Analyse-it V 4.8 and Microsoft excel.

\section{RESULTS}

Total 23 babies were studied. Among them, 11 were randomised to drug group and 12 to placebo group. The study was terminated before achieving the required sample size because of the inability to

Table 1. Clinical characteristics at recruitment

\begin{tabular}{|lrrr|}
\hline Quantitative variables - Median (IQR) & Drug group (N = 11) & Placebo group (N = 12) & P-value \\
\hline Birth weight (in grams) & & & \\
Gestation (in completed weeks) & $3000(2740-3466)$ & $3000(2400-3400)$ & 1.000 \\
Age at recruitment (in hours) & $38(37-38)$ & $38(37-39)$ & 0.726 \\
Mean systemic Blood pressure (mm Hg) & $8(7-15)$ & $8(1-12)$ & 1.000 \\
Oxygenation index & $51(42-55)$ & $46(38-53.8)$ & 0.548 \\
Right ventricular systolic pressure (mm Hg) & $18.6(16.1-24.8)$ & $21(18.7-26.4)$ & 1.000 \\
Right ventricular: systemic pressure & $41(33.8-56.3)$ & $54.5(44.7-64)$ & 0.343 \\
Fractional shortening (\%) & $0.96(0.6-1.27)$ & $1.2(0.83-1.64)$ & 0.343 \\
Qualitative variables (in percentage) & $33(26.7-40)$ & $35.4(30.2-48.3)$ & 1.000 \\
Gender (Male / Female) & & & \\
Right to left foetal shunt & $48.4 / 51.6$ & $58.3 / 41.7$ & 0.641 \\
Metabolic acidosis & 72.7 & 91.6 & 0.243 \\
Systemic hypotension & 45.4 & 41.6 & 0.857 \\
Meconium aspiration syndrome & 25 & 53.8 & 0.168 \\
Birth asphyxia & 72.2 & 75 & 0.881 \\
\hline
\end{tabular}

The P-value for the quantitative variable is calculated using the Sign test and that for the qualitative variable is calculated using the Chi-square test 


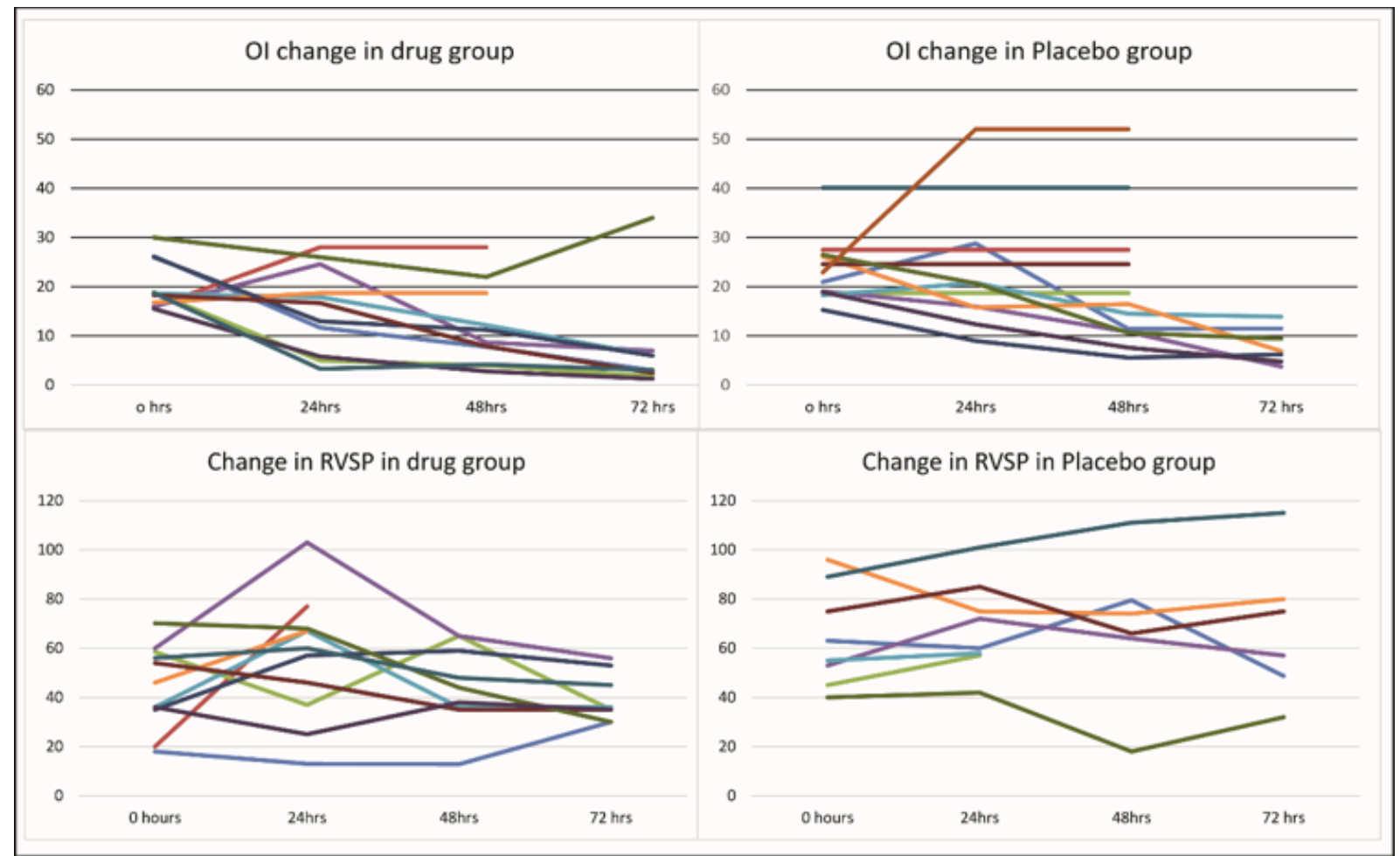

Figure 2. Graph showing the trend of OI and RVSP during the study period in both the groups

recruit more subjects and the extended study period also elapsed. Post hoc calculation of power using the actual sample size was $72.5 \%$

Median birth weight in both the groups was 3000 grams, gestation was 38 weeks, and age at recruitment was eight hours of life. Baseline characteristics are depicted in table 1 .

The oxygenation index (OI) was measured at least twice during the study period. Nine out of $11(82 \%)$ in the drug group had four measurements. Three of $11(27.2 \%)$ had an increase in the OI compared to their baseline. The Median (IQR) drop of OI from the baseline was $76.9 \%(-0.04$ to 88.2$)$. Seven out of 12 in the placebo group (58.3\%) subjects had four measurements. One subject had OI increased from the baseline (Figure 2). The Median (IQR) decrease in OI in the placebo group was $45.2 \%(0$ to 72). The difference in the drop of OI between both the group was not statistically significant ( $p=$ 0.548). Right ventricular systolic pressure (RVSP) was measured at least twice during the study period. Nine out of 11 subjects in the drug group had four measurements. Four (36.3\%) subjects had a higher RVSP compared to their baseline. The Median (IQR) decrease in RVSP in the drug group was $2.8 \%$ (- 50 to 32.6 ). In the placebo group, seven out of $12(58.3 \%)$ subjects had four measurements of RVSP. Three (25\%) subjects in this group had their RVSP elevated compared to their baseline (Figure 2).

Table 2. Comparison of complications between both groups

\begin{tabular}{|c|c|c|c|c|c|}
\hline & $\begin{array}{l}\text { Drug group } \\
\text { (\%) }\end{array}$ & $\begin{array}{l}\text { Placebo group } \\
(\%)\end{array}$ & $P$ value & $\begin{array}{l}\text { Relative Risk } \\
\text { (95\% CI) }\end{array}$ & $\begin{array}{c}\text { Number Needed } \\
\text { To treat }\end{array}$ \\
\hline Systemic hypotension & 81.8 & 83.3 & 0.923 & $0.98(0.67-1.4)$ & 66 (Benefit) \\
\hline Need for muscle relaxation & 72.8 & 91.7 & 0.256 & $0.79(0.53-1.18)$ & 5.3 (Benefit) \\
\hline Sepsis & 36.3 & 58.3 & 0.223 & $0.57(0.23-1.40)$ & 3.7 (Benefit) \\
\hline NEC & 9 & 8.3 & 0.948 & $1.09(0.07-15.4)$ & 132 (harm) \\
\hline Pneumothorax & 9 & 16.6 & 0.598 & $0.54(0.05-5.2)$ & 13.2 (Benefit) \\
\hline
\end{tabular}




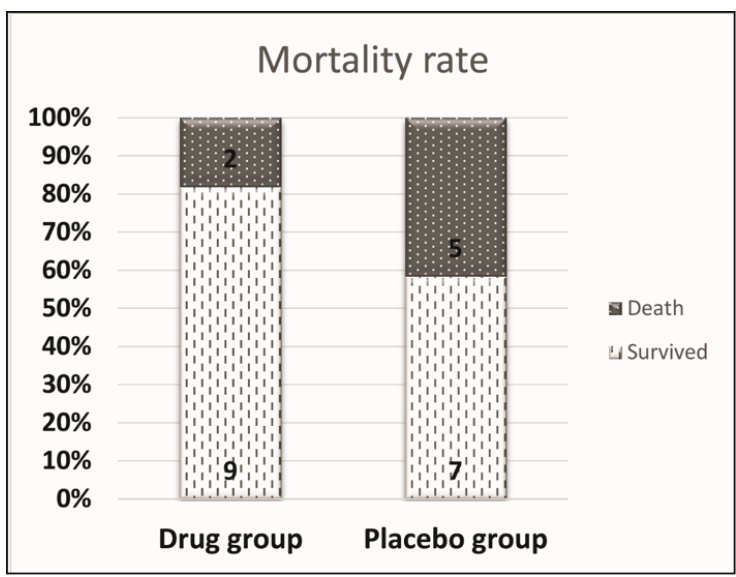

Figure 3. Survival rates in both the groups

Median (IQR) drop in RVSP in this group was $0 \%$ (- 6 to 14.2). The difference in the reduction of RVSP between the drug and the placebo group was not statistically significant $(\mathrm{p}=1.000)$. Babies who received the drug were ventilated for a median (IQR) of eight $(4.3$ - 9.8) days. Babies who received a placebo were ventilated for a median (IQR) of $10(9.2-11)$ days. This difference was not statistically significant $(p=0.218)$. Feeds were initiated at a median (IQR) of six (6 to 6) days in the drug group. In the placebo group, it was four (4 to 4) days. This difference was not statistically significant $(\mathrm{p}=1.000)$.

Full feeds were achieved in the drug group by median (IQR) 10.5 (7.9 to 14.2) in the placebo group, the median (IQR) age at the achievement of full feeds was 10 (9 to 11) days. This difference was not statistically significant $(\mathrm{p}=1.000)$. Sepsis, NEC, and pneumothorax were the complications encountered in our subjects (Table 2).

None of our subjects had pulmonary haemorrhage or developed intracranial haemorrhage. Two of the subjects in the drug group received surfactant which is a deviation from the protocol. This decision was based on the clinical judgment of the treating clinician. The Median (IQR) duration of hospital stay in the drug group was $13(10-15)$ days. In the placebo group, it was $13(2-20)$ days. The maximum duration of stay in the drug group was 30 days and that in the placebo group was 25 days. This difference was not statistically significant $(\mathrm{p}=1.000)$. Two babies $(18 \%)$ died in the drug group. Five (41.6\%) babies died in the placebo group (Figure 3).

The relative risk in the intervention group is 0.436 with a $95 \%$ CI between 0.105 and 1.80 . With this relative risk, the number needed to treat to benefit one in the drug group was 4.3 patients $\{95 \% \mathrm{CI}$ 7.74 (Harm) to 1.67 (Benefit) $\}$. The difference in the mortality rates between the drug and placebo groups was not statistically significant with a $\mathrm{p}$ value of 0.252 . After discharge, all the babies were followed up either in the developmental clinic or telephonic contact. All of them were alive and healthy at two years of age.

\section{DISCUSSION}

This study was undertaken to know the effectiveness of intravenous Sildenafil in comparison to placebo in reducing the mortality in 23 term babies on a ventilator with PPHN. Our study did not show any improvement in mortality rate in babies who received Sildenafil.

PPHN is a life-threatening neonatal pathology resulting from abnormal hemodynamic and respiratory adaptation to extrauterine life. Although iNO and ECMO are the mainstay of treatment of severe PPHN, they are expensive and unavailable in most of the centres. Less expensive alternatives such as Sildenafil have been studied for reducing PVR in symptomatic babies with PPHN. Several studies have been undertaken in the last decade to establish the role of IV Sildenafil in the management of PPHN. In the present study, the median (IQR) drop of OI from the baseline was $76.9 \%$ in the drug group whereas it was $45.2 \%$ in the placebo group. The difference in the drop of OI between both the group was not statistically significant $(\mathrm{p}=0.548)$.

In a study done by Steinhorn in 36 neonates to evaluate the efficacy of IV Sildenafil, a significant improvement in OI (28.7 to $19.3 ; \mathrm{P}=0.0002)$ was observed after four hours of Sildenafil infusion in the higher dose cohorts. 29 of these babies also received iNO. The measurement of OI was done after four hours of IV Sildenafil infusion which was a short-term response..$^{10}$ In contrast to this study, we studied the effect on OI for 72 hours which suggested the initial improvement in OI is not sustained. 
The Median (IQR) drop in RVSP in the drug group was $2.8 \%$ while in the placebo group it was $0 \%$. The difference in the drop of RVSP between the drug and the placebo group was not statistically significant $(\mathrm{p}=1.000)$. A similar result was reported by Vargas - Origel while comparing oral Sildenafil with placebo, where there was no significant difference in pulmonary arterial pressures between sildenafil and control (mean difference $1.10,95 \%$ CI -7.68 to 9.88$).{ }^{11}$ Oral Sildenafil absorption is variable in sick neonates. Our study has shown that even IV infusion which overcomes the absorption barrier did not have any significant effect on RVSP.

There was no significant difference in the number of ventilated days between the two groups in our study. This is in contrast to the study done in Turkey comparing Sildenafil with magnesium sulphate where a decrease in ventilation days among the Sildenafil group was demonstrated. ${ }^{12}$ Magnesium sulphate is known to cause sedation and muscle relaxation which could have contributed to prolonged ventilation in this study. This explains the apparent reduction in ventilation days may not be due to Sildenafil per se. All-cause mortality in the drug group was half of that in the placebo group. However, the difference was not statistically significant. A study done by Herrera reported the effect of Sildenafil on all-cause mortality like ours. ${ }^{13}$ However, the randomisation method was not described in this study by the authors. Another study was conducted by Vargas Origel in 51 babies with OI $>20$ and RVSP $>40$ using oral Sildenafil against placebo or iNO. They noted a significant decrease in all-cause mortality in the Sildenafil group only if the baseline OI was $>$ 40 and not otherwise. ${ }^{11}$ It is curious to note that the babies in this study were clinically sicker than our study (OI of 20 against OI of 15) and they successfully tolerated oral Sildenafil. Our study essentially removed the barrier of absorption to achieve a definitive systemic availability of the drug and yet did not find it effective in reducing the mortality. Partly this might be because of the lower sample size we could recruit. The rate of complications such as hypotension, NEC, sepsis, pneumothorax etc. was similar in both the groups. Intervention was neither beneficial nor harmful with respect to complications compared to placebo group. All the studies mentioned earlier, have looked into the short-term effect of Sildenafil as their primary outcome. To the best of our knowledge, this is the first study which has investigated all-cause mortality as primary outcome with Sildenafil therapy.

All the study subjects in our study were followed up and were found to be doing well. In a study done by Baqueroto know the outcome of term newborns treated with oral sildenafil for PPHN, four out of the seven subjects were followed up to 18 months. Neurodevelopment along with hearing assessments and MRI were normal in all of them. ${ }^{14}$ We believe that the lower power of the study due to the low sample size has contributed to the statistical insignificance of the difference in mortality rate. Although our study is a small sized study, we are hopeful that our study design and meticulous methodology should provide sufficient strength for our study to be cited in the future for similar studies.

\section{CONCLUSIONS}

The results from our study showed that Sildenafil did not reduce the mortality in ventilated babies with PPHN compared to placebo. There was also no significant difference in the duration of ventilation, OI, pulmonary pressure, complications or long-term outcome with this drug. Similar study with large sample size and longer duration of follow up might be helpful for general recommendation of use of Sildenafil in PPHN.

\section{REFERENCES}

1. Writing Committee Members, McLaughlin VV, Archer SL, Badesch DB, Barst RJ, Farber HW, et al. ACCF/AHA 2009 Expert Consensus Document on Pulmonary Hypertension: A Report of the American College of Cardiology Foundation Task Force on Expert Consensus Documents and the American Heart Association: Developed in 
Collaboration With the American College of Chest Physicians, American Thoracic Society, Inc., and the Pulmonary Hypertension Association. Circulation. 2009;119(16):2250-94. DOI:10.1161/CIRCULATIONAHA.109.192230

2. Walsh-Sukys MC, Tyson JE, Wright LL, Bauer CR, Korones SB, Stevenson DK, et al. Persistent pulmonary hypertension of the newborn in the era before nitric oxide: practice variation and outcomes. Pediatrics. 2000;105(1 Pt 1):14-20. DOI:10.1542/peds.105.1.14

3. Nakwan N, Jain S, Kumar K, Hosono S, Hammoud M, Elsayed YY, et al. An Asian multicenter retrospective study on persistent pulmonary hypertension of the newborn: incidence, etiology, diagnosis, treatment and outcome. J Matern Fetal Neonatal Med. 2020;33(12):2032-7. DOI:10.1080/14767058.2018.1536740

4. Sharma V, Berkelhamer S, Lakshminrusimha S. Persistent pulmonary hypertension of the newborn. Matern Health Neonatol Perinatol. 2015;1:14. DOI:10.1186/s40748-015-0015-4

5. Nair J, Lakshminrusimha S. Update on PPHN: Mechanisms and treatment. Semin Perinatol. 2014;38(2):78-91. DOI: $10.1053 /$ j.semperi.2013.11.004

6. Sildenafil for pulmonary hypertension in neonates - Kelly, LE - 2017 | Cochrane Library // www.cochranelibrary.com/cdsr/doi/10.1002/14651858.CD005494.pub4/abstract

7. Steinhorn RH. Neonatal Pulmonary Hypertension. Pediatr Crit Care Med J Soc Crit Care Med World Fed Pediatr Intensive Crit Care Soc. 2010;11(2):79-84. DOI:10.1097/PCC.0b013e3181c76cdc

8. Yaseen H, Darwich M, Hamdy H. Is Sildenafil an Effective Therapy in the Management of Persistent Pulmonary Hypertension? J Clin Neonatol. 2012;1(4):171-5. DOI: 10.4103/2249-4847.105958

9. Lakshminrusimha S, Mathew B, Leach CL. Pharmacologic Strategies in Neonatal Pulmonary Hypertension other than Nitric Oxide. Semin Perinatol. 2016;40(3):160-73. DOI: 10.1053/j.semperi.2015.12.004

10. Steinhorn RH, Kinsella JP, Pierce C, Butrous G, Dilleen M, Oakes M, et al. Intravenous sildenafil in the treatment of neonates with persistent pulmonary hypertension. J Pediatr. 2009;155(6):841-7. DOI: 10.1016/j.jpeds. 2009.06.012

11. Vargas-Origel A, Gómez-Rodríguez G, Aldana-Valenzuela C, Vela-Huerta MM, Alarcón-Santos SB, AmadorLicona N. The Use of Sildenafil in Persistent Pulmonary Hypertension of the Newborn. Am J Perinatol. 2010;27(03):225-30. DOI: 10.1055/s-0029-1239496

12. Uslu S, Kumtepe S, Bulbul A, Comert S, Bolat F, Nuhoglu A. A Comparison of Magnesium Sulphate and Sildenafil in the Treatment of the Newborns with Persistent Pulmonary Hypertension: A Randomised Controlled Trial. J Trop Pediatr. 2011;57(4):245-50. DOI:10.1093/tropej/fmq091

13. Torres RH, González PC, Castillo JH, Gutiérrez RGL, Balderrama IR. Oral sildenafil as an alternative treatment in the persistent pulmonary hypertension in newborns. Rev Mex Pediatría. 2006;73(4):159-63.

14. Baquero H, Neira F, Venegas ME, Valencia P, Sola A, Soliz A. 384 Outcome at 18 Months of Age After Sildenafil Therapy for Refractory Neonatal Hypoxemia. Pediatr Res. 2005;58(2):420-5. DOI: $10.1203 / 00006450-200508000-00413$ 